\title{
MEASURES ON $C(Y)$ WHEN $Y$ IS A COMPACT METRIC SPACE
}

\author{
JAMES KUELBS ${ }^{1}$
}

1. Introduction. Let $Y$ be a compact metric space and $C(Y)$ the space of real-valued continuous functions on $Y$ with the uniform topology. The minimal sigma-algebra containing the closed subsets of $C(Y)$ will be called the Borel subsets of $C(Y)$. If $F(p(1, \cdots, k))$, $p_{i} \in Y$, (where $p(1, \cdots, k)$ stands for $p_{1}, \cdots, p_{k}$ ) is a consistent family of finite-dimensional Gaussian distributions then sufficient conditions are given to assure the existence of a measure $\mu$ on the Borel subsets of $C(Y)$ whose finite-dimensional distributions are those postulated. It will also be shown that such a family of distributions always exists and that Levy's Brownian motion with parameter from the Hilbert space $l_{2}[1$, pp. 293-298] can be taken to have continuous sample paths when the parameter is restricted to certain compact subsets of $l_{2}$.

2. The product space $X=\prod_{k=1}^{\infty}\left[0,1 / 2^{k}\right]$ is assumed to have the topology induced by the usual $l_{2}$ norm $\|\cdot\|$. Let $F(p(1, \cdots, k)$ ), $p_{i} \in Y$, be a consistent family of Gaussian distributions. If for some homeomorphism $\phi$ of $Y$ into $X$ there exists another consistent family of Gaussian distributions $G(q(1, \cdots, k)), q_{i} \in X$, with the property that $F(p(1, \cdots, k))=G(\phi(p(1, \cdots, k)))$ (where $\phi(p(1, \cdots, k))$ stands for $\phi\left(p_{i}\right), \cdots, \phi\left(p_{k}\right)$ and

$$
\iint_{R_{2}}\left|s_{2}-s_{1}\right|^{2} d G(q(1,2))\left(s_{1}, s_{2}\right) \leqq\left. C\left\|q_{2}-q_{1}\right\|\right|^{\alpha}
$$

where $C>0, \alpha>0$ are independent of $q_{1}, q_{2}$ in $X$, then we say the family $F(p(1, \cdots, k)), p_{i} \in Y$, can be extended to $X$.

THEOREM 1. If $Y$ is a compact metric space and $F(p(1, \cdots, k))$, $p_{i} \in Y$, is a consistent family of Gaussian distributions which can be extended to $X$, then there exists a measure $\mu$ on the Borel subsets of $C(Y)$ whose finite-dimensional distributions are those given.

Corollary. If $Y=\prod_{k=1}^{\infty}\left[a_{k}, a_{k}+1 / 2^{k}\right]$ where $\left\{a_{k}\right\}$ is in $l_{2}$ and

Received by the editors November 3, 1965.

1 This research was supported by a National Science Cooperative Fellowship at the University of Minnesota and is a portion of the author's doctoral dissertation under Professor R. H. Cameron. Additional work on it was done at the University of Wisconsin. 
$F(p(1, \cdots, k)), p_{i} \in Y$, is the family of Gaussian distributions with mean vector zero and covariance matrix $V=\left(v_{i j}\right)$ where $2 v_{i j}=\left\|p_{i}\right\|+\left\|p_{j}\right\|$ $-\left\|p_{i}-p_{j}\right\|$ for $i, j=1, \cdots, k,[1$, p. 293], then there exists a measure $\mu$ on $C(Y)$ whose finite-dimensional distributions agree with those given.

Proof. Let $\phi\left(\left(y_{1}, y_{2}, \cdots\right)\right)=\left(y_{1}-a_{1}, y_{2}-a_{2}, \cdots\right)$. Then $\phi$ is a homeomorphism of $Y$ on to $X$. Let $G(q(1, \cdots, k))=F(p(1, \cdots, k))$ where $p_{i}=\phi^{-1}\left(q_{i}\right)$ for $i=1, \cdots, k$. Then

$$
\begin{aligned}
\iint_{R_{2}}\left|s_{2}-s_{1}\right|^{2} d G(q(1,2))\left(s_{1}, s_{2}\right) \\
=\iint_{R_{2}}\left|t_{2}-t_{1}\right|^{2} d F(p(1,2))\left(t_{1}, t_{2}\right)=\left\|p_{1}-p_{2}\right\|
\end{aligned}
$$

and the family $F(p(1, \cdots, k)), p_{i} \in Y$, can be extended to $X$ with respect to $\phi$. Hence the corollary follows from the previous theorem.

As a result of this corollary we see that Levy's Brownian motion with parameter from the Hilbert space $l_{2}$ can be defined to have continuous sample paths on subsets of the form $\prod_{k=1}^{\infty}\left[a_{k}, a_{k}+1 / 2^{k}\right]$. In fact, the conclusions hold if $Y$ is any compact subset of such a set. However, it is known [1, p. 293] that sample function continuity does not hold for arbitrary subsets of $l_{2}$.

3. The proof of our theorem will be obtained from the following sequence of lemmas. Throughout the discussion it is assumed that $X$ is as defined above and that $C(X)$ is the space of real-valued continuous functions on $X$ with the uniform topology. Since $X$ is compact $C(X)$ is a complete separable metric space and the results of Prokhorov [2] can be applied.

The Borel sets $S$ of $C(X)$ will be the minimal sigma-algebra containing the closed sets $\mathcal{F}$ of $C(X)$. The space of all probability measures on the measurable space $(C(X), S)$ will be denoted by $\mathfrak{T}(C(X))$. If $\left\{m_{k}\right\}$ is a sequence of elements in $\mathscr{T}(C(X))$ we say $\left\{m_{k}\right\}$ converges weakly to $m$ in $\mathfrak{T}(C(X))$ provided that

$$
\lim _{n \rightarrow \infty} \int_{C(X)} F(f) d m_{n}=\int_{C(X)} F(f) d m
$$

for every bounded continuous functional $F$ on $C(X)$. A metric is defined on $\mathfrak{T}(C(X))$ in the following way. If $m_{1}$ and $m_{2}$ are in $\mathfrak{T}$ we let $L\left(m_{1}, m_{2}\right)=\max \left\{\delta_{1}, \delta_{2}\right\}$ where

$$
\begin{array}{ll}
\delta_{1}=\inf \left\{\epsilon>0: m_{1}(F) \leqq m_{2}\left(F^{\epsilon}\right)+\epsilon\right. & \text { for all } F \in \mathcal{F}\}, \\
\delta_{2}=\inf \left\{\epsilon>0: m_{2}(F) \leqq m_{1}\left(F^{\epsilon}\right)+\epsilon\right. & \text { for all } F \in \mathcal{F}\}, \text { and }
\end{array}
$$




$$
F^{\boldsymbol{\epsilon}}=\left\{f \in C(X): \max _{x \in X}|f(x)-g(x)|<\epsilon \quad \text { for some } g \in F\right\} .
$$

In $[2$, pp. 168-170] it is shown that $L$-convergence and weak convergence are equivalent in $\mathscr{T}(C(X))$ and that $\mathscr{T}(C(X))$ is a complete separable metric space in these topologies. If $\theta$ is a continuous mapping of $C(X)$ into $C(X)$ and $m \in \mathscr{M}(C(X))$ we will denote by $m^{\theta}$ the element of $\mathfrak{T C}(C(X))$ such that $m^{\theta}(A)=m\left(\theta^{-1}(A)\right)$ for all $A \in S$. If $\delta>0$ and $m(H)>1-\epsilon$, where

$$
H=\left\{f \in C(X): \max _{x \in X}|f(x)-\theta(f)(x)|<\delta\right\},
$$

then $L\left(m, m^{\theta}\right) \leqq \max (\epsilon, \delta)$. This estimate is essential to us; its proof is in $[2$, p. 167].

Lemma 1. Let $H=\prod_{j=1}^{N}\left[a_{j}, b_{j}\right]$ where $-\infty<a_{j}<b_{j}<\infty$ for $j$ $=1, \cdots, N$ and suppose $f\left(x_{1}, \cdots, x_{N}\right)$ is a real-valued function on $H$. Then there exists a continuous function $\hat{f}$ on $H$ such that $\hat{f}=f$ on $\mathcal{P}=\left\{\left(x_{1}, \cdots, x_{N}\right): x_{j}=a_{j}\right.$ or $\left.x_{j}=b_{j}\right\}$ and $\max _{p \in H} \hat{f}(p)=\max _{p \in \mathcal{P}} \hat{f}(p)$ $\geqq \min _{p \in \mathcal{P}} \hat{f}(p)=\min _{p \in I I} \hat{f}(p)$. In fact, we take $\hat{f}$ to be as follows:

$$
\begin{aligned}
\hat{f}\left(x_{1}, \cdots, x_{N}\right)= & \sum_{i=1}^{2^{N}} f\left(y_{1}^{i}, \cdots, y_{N}^{i}\right) \\
& \frac{\left(x_{1}-a_{1}\right)^{2_{1}^{i}}\left(b_{1}-x_{1}\right)^{1-i 1} \cdots\left(x_{N}-a_{N}\right)^{z_{N}^{i}}\left(b_{N}-x_{N}\right)^{1-z_{N}^{i}}}{\left(b_{1}-a_{1}\right) \cdots\left(b_{N}-a_{N}\right)}
\end{aligned}
$$

where $\left\{\left(y_{1}^{i}, \cdots, y_{N}^{i}\right): i=1, \cdots, 2^{N}\right\}=\mathcal{P}, z_{j}^{i}=0$ if $y_{j}^{i}=a_{j}, z_{j}^{i}=1$ if $y_{j}^{i}=b_{j}$, and we assume $\left(x_{j}-a_{j}\right)^{0}=\left(b_{j}-x_{j}\right)^{0} \equiv 1$.

Proof. It is clear that $\hat{f}$ is continuous on $H$ and that $\hat{f}=f$ on $P$. The proof that $\max _{p \in H} \hat{f}(p)=\max _{p \in \mathcal{P}} \hat{f}(p) \geqq \min _{p \in \mathcal{P}} \hat{f}(p)=\min _{p \in H} \hat{f}(p)$ follows by induction on $N$.

Let $\rho_{N}=\left\{\left(x_{1}, \cdots, x_{N}, 0, \cdots\right): x_{k}=j / 2^{N}, 0 \leqq j \leqq 2^{N-k}\right\}$ for $N=1,2, \cdots$. The number of points of $X$ which $\rho_{N}$ contains will be denoted by $\operatorname{ord}\left(\odot_{N}\right)$. Hence we have ord $\left(\odot_{N}\right) \leqq 2^{N(N+1) / 2}$. Let

$$
H_{N}=\prod_{k=1}^{N}\left[0,1 / 2^{k}\right], \quad \text { and } H_{N}^{i}=\prod_{k=1}^{N}\left[a_{k}^{i}, a_{k}^{i}+1 / 2^{N}\right]
$$

where $a_{\boldsymbol{k}}^{i}=j / 2^{N}$ and $j$ is an integer depending on $i$ and $k$ such that $0 \leqq j \leqq 2^{N-k}-1$ for $k=1, \cdots, N$. Then there are $2^{N(N-1) / 2}$ distinct $H_{N}^{i}$ and by relabeling the $H_{N}^{i}$, if necessary, we have $H_{N}$ as the union of the $H_{N}^{i}$ for $1 \leqq i \leqq 2^{N(N-1) / 2}$. Moreover, the $H_{N}^{i}$ overlap only on 
their boundaries when they are considered as subsets of $R_{N}$. If $f$ is any real-valued function on $X$ we construct the function $\hat{f}_{N}$ on $X$ such that $\hat{f}_{N}\left(x_{1}, \cdots, x_{N}, x_{N+1}, \cdots\right)=\hat{f}_{i}\left(x_{i}, \cdots, x_{N}, 0,0, \cdots\right)$ for $\left(x_{1}, \cdots, x_{N}, 0, \cdots\right)$ in $H_{N}^{i} \times(0,0, \cdots)$ and where $\hat{f}_{i}\left(x_{1}, \cdots, x_{N}, 0, \cdots\right)$ is defined on $H_{N}^{i} \times(0,0, \cdots)$ by using Lemma 1 on $f\left(x_{1}, \cdots, x_{N}, 0, \cdots\right)$ and the obvious homeomorphism. Then $\hat{f}_{N}$ is continuous on $X$ and $\hat{f}_{N}=f$ on $\mathcal{P}_{N}$. Moreover, the maximum and minimum of $\hat{f}_{N}$ over $X$ are both attained on $\rho_{N}$.

Definition. If $f$ is in $C(X)$ let $G_{N}(f)=\hat{f}_{N}$ where $\hat{f}_{N}$ is defined as above. Let $S_{N}=G_{N}(C(X))$ for $N=1,2, \cdots$.

From the above considerations it is quite clear that $G_{N}$ is a con tinuous mapping of $C(X)$ onto the closed linear subspace $S_{N}$ of $C(X)$ and that $S_{N}$ is homeomorphic to $R_{L}$ where $L=\operatorname{ord}\left(\odot_{N}\right)$. Let $G(q(1, \cdots, k))$, $q_{i} \in X$, be a system of consistent Gaussian distributions. If $E$ is any Borel subset of $R_{L}$ and $I=\left\{f \in S_{N}:\left[f\left(q_{1}\right), \cdots, f\left(q_{L}\right)\right] \in E\right\}$ where $\left\{q_{1}, \cdots, q_{L}\right\}=\mathcal{P}_{N}$ then we define $m_{N}(I)=\int_{E} d G(q(1, \cdots, L))$. Then $m_{N}$ is a measure on the Borel subsets of $S_{N}$ and since $S_{N}$ is a closed subset of $C(X)$ we can extend $m_{N}$ to a measure on the Borel subsets of $C(X)$ simply by letting $m_{N}\left(C(X)-S_{N}\right)=0$. That is, $m_{N}$ so extended is an element of $\mathfrak{T}(C(X))$. Moreover, if $\theta=G_{N-1}$ then $m_{N}^{\theta}=m_{N-1}$ for $N=2,3, \cdots$.

Lemma 2. The sequence $\left\{m_{N}\right\}$ is a Cauchy sequence in $\operatorname{mr}(C(X))$ provided

$$
\iint_{R_{2}}\left|s_{2}-s_{1}\right|^{2} d G(q(1,2))\left(s_{1}, s_{2}\right) \leqq C\left\|q_{1}-q_{2}\right\|^{\alpha}
$$

for all $q_{1}, q_{2}$ in $X$ where $C>0$ and $\alpha>0$ are constants independent of $q_{1}, q_{2}$ and $\left\|q_{1}-q_{2}\right\|$ is the usual $l_{2}$ distance between $q_{1}, q_{2}$.

Proof. We will examine $L\left(m_{N}, m_{N-1}\right)$ by noting that $L\left(m_{N}, m_{N-1}\right)$ $=L\left(m_{N}, m_{N}^{\theta}\right)$ where $\theta=G_{N-1}$. Since $m_{N}\left(C(X)-S_{N}\right)=0$ we have

$$
\begin{aligned}
J & =m_{N}\left\{f \in C(X): \max _{a \in X}|\theta(f)(q)-f(q)|>1 / N^{2}\right\} \\
& =m_{N}\left\{f \in S_{N}: \max _{q \in X}|\theta(f)(q)-f(q)|>1 / N^{2}\right\} .
\end{aligned}
$$

Now if $f \in S_{N}$ then $\theta(f)$ is in $S_{N-1}$ and

$$
\max _{\boldsymbol{a} \in X}|f(q)-\theta(f)(q)|=\max _{1 \leqq i \leqq K}\left[\max _{\boldsymbol{q} \in Z_{i}}|f(q)-\theta(f)(q)|\right]
$$

where $K=2^{(N-1)(N-2) / 2}$ and $Z_{i}=H_{N-1}^{i} \times\left[0,1 / 2^{N}\right] \times(0, \cdots)$. Furthermore, if $f \in S_{N}$ Lemma 1 implies that 


$$
\max _{\boldsymbol{q} \in Z_{\mathbf{i}}}|f(q)-\theta(f)(q)| \leqq\left|f\left(q_{1}\right)-f\left(q_{2}\right)\right|
$$

where $q_{1} \in \mathcal{P}_{N} \cap Z_{i}, q_{2} \in \mathcal{P}_{N-1} \cap Z_{i}$, and $q_{1} \neq q_{2}$ depend on $f$. Hence

$$
J \leqq \sum_{i=1}^{K} m_{N}\left\{f \in S_{N}: \max _{g \in Z_{i}}|f(q)-\theta(f)(q)|>1 / N^{2}\right\}
$$

and we see that

$$
\begin{aligned}
m_{N}\left\{f \in S_{N}: \max _{q \in Z_{i}} \mid f(q)\right. & \left.-\theta(f)(q) \mid>1 / N^{2}\right\} \\
& \leqq \sum_{\left(q_{1}, q_{2}\right) \in A} m_{N}\left\{f \in S_{N}:\left|f\left(q_{1}\right)-f\left(q_{2}\right)\right|>1 / N^{2}\right\}
\end{aligned}
$$

where $A=\left\{\left(q_{1}, q_{2}\right): q_{1} \in \mathcal{P}_{N} \cap Z_{i}, q_{2} \in \mathcal{P}_{N-1} \cap Z_{i}, q_{1} \neq q_{2}\right\}$. Now $\left(q_{1}, q_{2}\right)$ $\in A$ implies $\left\|q_{1}-q_{2}\right\|<N^{1 / 2} / 2^{N-1}$ so for $\left(q_{1}, q_{2}\right) \in A$ and $\gamma$ a positive integer we have

$$
\begin{aligned}
m_{N}\left\{f \in S_{N}: \mid f\left(q_{1}\right)\right. & \left.-f\left(q_{2}\right) \mid>1 / N^{2}\right\} \\
& =m_{N}\left\{f \in S_{N}:\left|f\left(q_{1}\right)-f\left(q_{2}\right)\right|^{2 N \cdot \gamma}>\left(1 / N^{2}\right)^{2 N \gamma}\right\} \\
& \leqq N^{4 N \gamma} \int_{S_{N}}\left|f\left(q_{1}\right)-f\left(q_{2}\right)\right|^{2 N \gamma} d m_{N} \\
& =N^{4 N \gamma}\left[\operatorname{Var}\left(f\left(q_{1}\right)-f\left(q_{2}\right)\right)\right]^{N \gamma}[1 \cdot 3 \cdot 5 \cdots(2 N \gamma-1)] \\
& \leqq C N^{4 N \gamma}\left[N^{1 / 2} / 2^{N-1}\right]^{N \gamma \alpha}[1 \cdot 3 \cdot 5 \cdots(2 N \gamma-1)] .
\end{aligned}
$$

Letting ord $(A)$ denote the number of elements of $A$ we have ord $(A)$ $\leqq 3^{N} \cdot 2^{N}$ and $K \cdot \operatorname{ord}(A) \leqq 2^{N^{2} / 2+3 N / 2+1}$. Hence

$$
J \leqq C \cdot 2^{N^{2} / 2+3 N / 2+1} \cdot N^{4 N \gamma}[1 \cdot 3 \cdot 5 \cdots(2 N \gamma-1)]\left[N^{1 / 2} / 2^{N-1}\right]^{N \gamma \alpha}
$$

and choosing $\gamma$ so that $\gamma \cdot \alpha \geqq 1$ we see that $J \leqq \exp \{-N \gamma\}$ for $N$ sufficiently large. As a result of the estimate [2, p. 167] we have $L\left(m_{N}, m_{N-1}\right) \leqq \max \left\{e^{-N \gamma}, N^{-2}\right\}$ for $N$ sufficiently large and hence $\left\{m_{N}\right\}$ is a Cauchy sequence in the $L$-metric so our proof is complete.

If $G(q(1, \cdots, k)), q_{i} \in X$, is a consistent family of Gaussian distributions satisfying the conditions of Lemma 2 then $\left\{m_{N}\right\}$ is a Cauchy sequence in $\mathscr{T}(C(X))$ and there exists an element $m$ in $\mathscr{T C} C(X)$ such that $\left\{m_{N}\right\}$ converges weakly to $m$. Moreover, $m_{N}{ }^{G}$ will converge weakly to $m^{G_{M}}$ and since $m_{N}{ }^{G_{M}}=m_{M}$ for $N \geqq M$ we have $m^{G_{M}}=m_{M}$. That is, if $\left\{q_{1}, \cdots, q_{k}\right\} \subseteq \odot_{N}$ for some $N$ then

$$
m\left\{f \in C(X):\left[f\left(q_{1}\right), \cdots, f\left(q_{k}\right)\right] \in E\right\}=\int_{E} d G(q(1, \cdots, k))
$$


for any Borel subset $E$ of $R_{k}$. To show that all the finite-dimensional distributions of $m$ coincide with those postulated follows quite easily.

The following theorem summarizes the results of this section.

Theorem 2. If $G(q(1, \cdots, k)), q_{i} \in X$, is a consistent family of Gaussian distributions then there exists a measure $m$ on the Borel subsets of $C(X)$ whose finite-dimensional distributions coincide with those postulated provided that for all $q_{1}, q_{2}$ in $X$

$$
\iint_{R_{2}}\left|s_{2}-s_{1}\right|^{2} d G(q(1,2))\left(s_{1}, s_{2}\right) \leqq C\left\|q_{1}-q_{2}\right\| \alpha
$$

where $C>0$ and $\alpha>0$ are independent of $q_{1}, q_{2}$.

4. Proof of Theorem 1. Since the family $F(p(1, \cdots, k))$, $p_{i} \in Y$, can be extended to $X$ there exists a consistent family of Gaussian distributions $G(q(1, \cdots, k)), q_{i} \in X$, which satisfies the conditions of Theorem 2 and $F(p(1, \cdots, k))=G(\phi(p(1, \cdots, k)))$ where $\phi$ is a homeomorphism of $Y$ into $X$. Let $m$ be the measure on $C(X)$ obtained as a result of Theorem 2. Since $\phi$ is a homeomorphism of $Y$ into $X$ we have $\theta(f)=f(\phi(\cdot))$ mapping $C(X)$ continuously on to $C(Y)$. Then $m^{\theta}$ is a measure on the Borel subsets of $C(Y)$ and if $I=\left\{g \in C(Y):\left[g\left(p_{1}\right), \cdots, g\left(p_{k}\right)\right] \in E\right\}$ where $p_{1}, \cdots, p_{k}$ are in $Y$ and $E$ is a Borel subset of $R_{k}$ we have

$$
\begin{aligned}
m^{\theta}(I) & =m\left(\theta^{-1}(I)\right) \\
& =m\left\{f \in C(X):\left[\theta(f)\left(p_{1}\right), \cdots, \theta(f)\left(p_{k}\right)\right] \in E\right\} \\
= & \int_{E} d G(\phi(p(1, \cdots, k)))=\int_{E} d F(p(1, \cdots, k)) .
\end{aligned}
$$

Hence $\mu=m^{\theta}$ has the finite-dimensional distributions postulated and the theorem is proved.

5. Since $Y$ is a compact metric space there exists a homeomorphism $\phi$ of $Y$ in to $X$. Consequently, if $G(q(1, \cdots, k)), q_{i} \in X$, is the family of distributions mentioned in the proof of the Corollary to Theorem 1 then the family $F(p(1, \cdots, k))=G(\phi(p(1, \cdots, k))), p_{i} \in Y$, satisfies Theorem 1 so the existence question mentioned in the introduction is easily handled.

As a final remark we mention that the conclusions of Theorem 2 hold if the hypotheses are such that the integral

$$
\iint_{R_{2}}\left|s_{2}-s_{1}\right|^{2} d G(q(1,2))\left(s_{1}, s_{2}\right)
$$


is less than or equal to $C\left|q_{1}-q_{2}\right|^{\alpha}$ where $C>0, \alpha>0$ are independent of $q_{1}, q_{2}$ and $\left|q_{1}-q_{2}\right|=\sum_{i=1}^{\infty}\left|b_{i}-a_{i}\right|$ when $q_{1}=\left(a_{1}, a_{2}, \cdots\right), q_{2}$ $=\left(b_{1}, b_{2}, \cdots\right)$.

\section{BIBLIOGRAPHY}

1. P. Levy, Processus stochastiques et mouvement brownien, Gauthier-Villars, Paris 1948, 275-298.

2. Yu. V. Prokhorov, Convergence of random processes and limit theorems in probability theory, Teor. Verojatnost. i Primenen. 1 (1956), 177-238 = Theor. Probability Appl. 1 (1956), 157-214.

3. I. J. Shoenberg, On cerlain melric spaces arising from euclidean spaces by a change of metric and their imbedding in Hilbert space, Ann. of Math. 38 (1937), 787-793.

UNIVERSITY OF WISCONSIN 